\title{
Inestabilidad laboral en el empleo, duración del desempleo y depreciación del capital humano*
}

\author{
Virginia Hernanz \\ Universidad de Alcalá de Henares \\ Juan Francisco Jimeno \\ Banco de España, CEPR, IZA**
}

\begin{abstract}
Resumen
La elevada incidencia del empleo temporal en España provoca que muchos trabajadores tengan trayectorias laborales con múltiples transiciones entre trabajos de corta duración y episodios de desempleo. La elevada rotación laboral entre diferentes puestos de trabajo se traduce en una menor acumulación de capital humano a la vez que los periodos de desempleo provocan una depreciación adicional del mismo que conlleva una menor probabilidad de salir del desempleo y una pérdida salarial en el nuevo empleo. En este artículo, a partir de datos de la Muestra Continua de Vidas Laborales (MCVL) del periodo 2006 y 2016, mostramos en qué medida la permanencia en el desempleo y el ajuste salarial en las posteriores salidas al empleo está relacionada con diferentes medidas de inestabilidad a lo largo de la carrera laboral de los individuos. Los resultados indican que una mayor acumulación de experiencia laboral como trabajador temporal, en lugar de como indefinido, está asociada con una menor probabilidad de salida del desempleo. En el caso de los salarios, las pérdidas salariales tras el paso por el desempleo son superiores cuanto mayor estabilidad previa hubiera acumulado el trabajador, probablemente reflejando la pérdida de capital humano específico tras el paso por el desempleo.
\end{abstract}

Palabras clave: desempleo, acumulación y depreciación del capital humano, salarios.

Clasificación JEL: J24, J31, J41, J63, J64.

\begin{abstract}
The high incidence of temporary employment in Spain causes many workers to have employment trajectories with multiple transitions between short-term jobs and unemployment spells. The high labour turnover between different jobs translates into a lower accumulation of human capital, while the periods of unemployment cause an additional depreciation that leads to a lower probability of leaving the unemployment and a wage loss in a new job. In this article, using data from the Continuous Sample of Working Life (MCVL) between 2006 and 2016, we show to what extent the unemployment duration and wage adjustments in the subsequent exits to employment is related to different measures of instability throughout the labour market career. The results indicate that a greater accumulation of work experience as a temporary worker, instead of as a permanent worker, is associated with a lower probability of leaving unemployment. In the case of wages losses, after passing through unemployment they are higher the more previous stability the worker had accumulated, probably reflecting the loss of specific human capital while unemployed.
\end{abstract}

Keywords: unemployment, human capital accumulation and depreciation, wages.

JEL classification: J24, J31, J41, J63, J64.

* Las opiniones y los análisis de este artículo son responsabilidad de los autores y, por tanto, no necesariamente coinciden con los del Banco de España o el Eurosistema.

** CEPR: Centre for Economic Policy Research; IZA: Institute of Labor Economics. 


\section{Introducción}

El mercado laboral español ha registrado un elevado desempleo desde principios de los años ochenta y una alta incidencia de empleos de corta duración desde la reforma que liberalizó los contratos temporales de trabajo en 1984. Como resultado, muchos trabajadores alternan periodos «inestables» de empleo (esto es, periodos de ocupación con transiciones entre varios puestos de trabajo) con periodos de desempleo que, a veces, son de larga duración.

Estas pautas de ocupación y paro tienen dos consecuencias sobre la acumulación de capital humano de los trabajadores. Por una parte, durante los periodos de ocupación, los trabajadores con empleos «más inestables» tienen menores ganancias de productividad por dos motivos. Uno es que, como se ha documentado en numerosos trabajos, los trabajadores con contrato temporal de trabajo reciben menos formación. En el caso español, Albert et al. (2004) documentan que los trabajadores con contratos temporales no solo tienen una menor probabilidad de ser contratados por empresas que ofrecen formación, sino que incluso, si son contratados por este tipo de empresas, también tienen una menor probabilidad de ser elegidos para participar en este tipo de actividades. Adicionalmente, Peraita (2005), a partir de datos del panel de hogares europeos para 1994, muestra que los trabajadores con menor nivel educativo también presentan una menor probabilidad de recibir formación y que, además, las instituciones laborales que contribuyen a estructuras salariales más comprimidas, como es el caso de España, restan incentivos a la inversión en formación por parte de las empresas. Más recientemente Cabrales et al. (2014) analizan cómo estas diferencias entre la formación que reciben los trabajadores temporales y los indefinidos se acrecientan en los países que experimentan una mayor segmentación del mercado de trabajo y muestran que tienen consecuencias duraderas sobre las diferencias de habilidades cognitivas entre trabajadores con trayectorias laborales más estables y aquellos que sufren la inestabilidad laboral.

Otro factor relevante en la acumulación de capital humano es el tipo de capital acumulado en función de experiencia laboral. En principio, cabe esperar que trabajadores ocupados en empleos diferentes (en ocasiones de sectores y ocupaciones distintas) obtengan mayores ganancias de capital humano específico a una empresa u ocupación que los trabajadores que mantienen empleos más duraderos en los mismos sectores y ocupaciones. No obstante, mientras que existen estimaciones sobre la magnitud de las ganancias de productividad por experiencia laboral a partir de ecuaciones de salarios a la Mincer (1974) ${ }^{1}$, no hay evidencia precisa sobre en qué medida la experiencia laboral produce rendimientos distintos en función de la estabilidad de la trayectoria laboral en cuestión.

En cuanto a la incidencia del desempleo sobre la depreciación del capital humano, existe también una extensa literatura que confirma que a mayor duración del

${ }^{1}$ Véase, por ejemplo, DE LA FUENTE y JIMENO (2009). 
desempleo mayor es la pérdida de habilidades laborales ${ }^{2}$. Sin embargo, aunque hay razones para pensar que dicha depreciación puede ser diferente en función de la trayectoria laboral en el empleo anterior al desempleo, no hay resultados empíricos que confirmen o refuten tal asociación. Por una parte, si los trabajadores que entran en el desempleo lo hacen tras episodios inestables de ocupación, es posible que solo sufran depreciación del capital humano general acumulado durante su etapa educativa, dado que no parecen recibir formación en el empleo intensivamente. Por otra, disponen de menos capital humano específico a una empresa u ocupación que, probablemente, es el que se deprecia a mayor ritmo durante el desempleo.

Con estas premisas, el objetivo de este trabajo es documentar la asociación entre inestabilidad del empleo en periodos de ocupación y tasas de salida y pérdida salarial en el reempleo en periodos posteriores al desempleo. Puesto que las tasas de salida del desempleo a distintas duraciones del mismo y los salarios con los que se entra en el nuevo empleo deben ser, en parte, reflejo del capital humano de los trabajadores que realizan dichas transiciones, esta asociación puede tomarse como un indicio de los efectos que la inestabilidad laboral tiene sobre la acumulación y la depreciación de capital humano y, por tanto, sobre la productividad de los trabajadores afectados por ella. La importancia de tales efectos en el largo plazo podría ser considerable. Por ejemplo, García-Pérez, Ionescu y Vall-Castello (2018) concluyen que la contratación temporal habría reducido el empleo de la cohorte que entró en el mercado de trabajo en el momento de su liberalización en 1984 en casi un año (hasta 2006) con una pérdida salarial acumulada del 12 por 100 .

Para ello, se construyen, en primer lugar, un conjunto de indicadores sobre el grado de inestabilidad que ha experimentado el trabajador en toda su vida laboral. En concreto, se calcula el número de empleos diferentes que ha tenido el trabajador a lo largo de su vida laboral, definido a partir del total de relaciones laborales observadas en la MCVL. Para disponer de una medida relativa de la frecuencia de cambios de empleo a lo largo de la vida laboral, se divide el total de empleos por los años de experiencia potencial de cada individuo. Asimismo, se ha calculado el número de días totales trabajados como porcentaje de la experiencia potencial del trabajador ${ }^{3}$ así como su distribución entre aquellos días trabajados con un contrato temporal y con un contrato indefinido. En segundo lugar, y tomando estos indicadores junto con el habitual grupo de controles en análisis de duración del desempleo y de los determinantes salariales, se estima la probabilidad de que estos trabajadores que han sido despedidos encuentren un trabajo a lo largo de los tres años siguientes y los salarios con los que se realizan dichas transiciones.

Los resultados muestran que existe una relación negativa estadísticamente significativa entre inestabilidad de trayectorias laborales en el empleo, por una parte, y tasas de salida del desempleo y salarios del nuevo empleo, por otra.

${ }^{2}$ Véanse, por ejemplo, MINCER y OFEK (1982), EDIN y GUSTAVSSON (2008), ARRAZOLA et al. (2005), GREGORY y JUKES (2001) y ARULAMPALAM (2001).

${ }^{3}$ Calculada como los días totales transcurridos desde el primer contrato de trabajo. 
El trabajo se estructura en cuatro apartados más. En el segundo apartado, se describen los datos utilizados y la construcción de variables y de la muestra a utilizar en las estimaciones de tasas de salida del desempleo y de salarios en el nuevo empleo. En los apartados tercero y cuarto se presentan las especificaciones de los modelos utilizados para realizar dichas estimaciones y los correspondientes resultados. Finalmente, el quinto apartado contiene comentarios y conclusiones finales.

\section{Datos y preparación de la muestra}

La fuente de datos es la Muestra Continua de Vidas Laborales (MCVL) en su versión extendida con datos fiscales. Esta muestra contiene la vida laboral de un 4 por 100 de trabajadores que mantuvieran una relación de alta laboral con la Seguridad Social. Para este trabajo, tomamos las distintas ediciones de la MCVL realizadas desde 2005 (para una información más detallada sobre el uso de la MCVL en el estudio de las trayectorias laborales, véase Arranz et al., 2013).

Para construir las observaciones con las que estimar la asociación entre inestabilidad de trayectorias laborales en el empleo y tasas y salarios de salida en periodos posteriores de desempleo, seleccionamos de la MCVL a los asalariados (temporales e indefinidos) que perdieron sus empleos en algún momento de los años 2006, 2010, 2012 y 2014, y que sufrieran posteriormente un periodo de desempleo de una duración mínima de tres meses ${ }^{4}$. De tales observaciones, se excluyen los asalariados a tiempo parcial puesto que la información disponible en la MCVL sobre horas trabajadas es incompleta y no permite calcular salarios comparables en periodos distintos de empleo. A partir de estos datos de la MCVL se ha creado un conjunto de variables que miden la duración del último episodio de desempleo del individuo, el número de empleos que ha tenido el individuo a lo largo de su vida laboral, sus antigüedades totales con empleos temporales e indefinidos y la proporción que cada una de ellas representa con respecto a la experiencia potencial (antigüedades relativas) que el trabajador hubiera podido llegar a tener si no hubiera dejado de trabajar desde que tuvo su primera alta laboral. Además, se computan el salario del último empleo previo al despido y el salario del primer empleo después del último episodio de desempleo ${ }^{5}$ observado para analizar el impacto de la pérdida de empleo sobre los salarios y en qué medida este impacto está relacionado con la experiencia laboral previa del trabajador y, en particular, con las medidas de rotación laboral antes mencionadas. En el análisis econométrico se incorporan como variables de control adicionales las variables personales y del puesto de trabajo habituales en este tipo de estudios y que se encuentran en la MCVL como la edad, nacionalidad, sexo, el tipo de contrato, grupo de cotización, sector de actividad y el tamaño de la empresa.

\footnotetext{
${ }^{4}$ Esta última elección intenta limpiar el análisis de aquellas entradas y salidas del empleo/desempleo de muy corta duración, que aunque también pueden conllevar pérdidas de capital humano, no son el objetivo principal de nuestro análisis.

5 En ambos casos, se utiliza el salario diario mensualizado deflactado para evitar comparaciones entre salarios mensuales con diferente número de días trabajados.
} 
La duración en el desempleo es una medida fundamental para poder analizar cómo ha ido modificándose la probabilidad de salida de los desempleados durante los años de observación y en función del tiempo transcurrido en el desempleo. La duración máxima en el desempleo observada en nuestra muestra son 36 meses, siguiendo el criterio establecido en otros trabajos previos, y dado que la probabilidad de salida observada se reduce y se vuelve prácticamente nula a partir de este límite. También se incluye como parte de la información relevante si los trabajadores despedidos están cobrando prestaciones, dada la importancia de las misma tanto para explicar la rotación (véase Jenkins y García-Serrano, 2004 y Rebollo, 2012) como para su posterior salida del desempleo (véase Rebollo y García-Pérez, 2015).

En el Cuadro 1, donde se describen las características básicas de nuestra muestra, se observa que el conjunto de trabajadores despedidos es relativamente joven, aunque la edad media va subiendo a lo largo del período considerado. En cuanto al sexo, la proporción de mujeres disminuye durante la crisis, reflejando el impacto de la crisis sobre sectores mayoritariamente masculinos como la construcción. También se observa una incidencia muy elevada de los despidos entre los trabajadores de nacionalidad extranjera, ya que su presencia es superior a la observada entre los empleados: los extranjeros solo representan alrededor de un 10 por 100 del empleo total y aproximadamente un 20 por 100 del total de despido. Las entradas en el desempleo también se producen de manera mayoritaria desde un contrato temporal,

\section{CUADRO 1}

PRINCIPALES ESTADÍSTICOS DESCRIPTIVOS

(Media y desviación de las variables dicotómicas que toman valor 1 en caso afirmativo y 0 en el contrario)

\begin{tabular}{|l|c|c|c|c|c|}
\hline \multicolumn{2}{|c|}{} & $\mathbf{2 0 0 6}$ & $\mathbf{2 0 1 0}$ & $\mathbf{2 0 1 2}$ & $\mathbf{2 0 1 4}$ \\
\hline \multirow{2}{*}{ Edad } & Media & 36,41 & 38,53 & 40,49 & 41,15 \\
\cline { 2 - 6 } & DT & 13,00 & 12,19 & 12,10 & 12,05 \\
\hline \multirow{2}{*}{ Sexo (mujeres) } & Media & 0,49 & 0,44 & 0,45 & 0,44 \\
\cline { 2 - 6 } & DT & 0,50 & 0,50 & 0,50 & 0,50 \\
\hline \multirow{2}{*}{ Temporales } & Media & 0,82 & 0,78 & 0,82 & 0,82 \\
\cline { 2 - 6 } & DT & 0,38 & 0,41 & 0,39 & 0,38 \\
\hline \multirow{2}{*}{ Meses sin empleo } & Media & 0,78 & 0,71 & 0,69 & 0,74 \\
\cline { 2 - 6 } & DT & 0,41 & 0,46 & 0,46 & 0,44 \\
\hline \multirow{2}{*}{ Cobrar prestaciones } & Media & 9,89 & 11,63 & 12,78 & 10,61 \\
\cline { 2 - 6 } & DT & 6,83 & 7,91 & 8,54 & 6,73 \\
\cline { 2 - 6 } & Media & 0,35 & 0,51 & 0,49 & 0,45 \\
\cline { 2 - 6 } & DT & 0,48 & 0,50 & 0,50 & 0,50 \\
\hline
\end{tabular}

FUENTE: MCVL.

NOTA: DT se utiliza como abreviatura de desviación típica. 
aunque durante la crisis el porcentaje de salidas desde un empleo indefinido aumentó considerablemente (lo que se refleja directamente en el aumento que experimentó el porcentaje de personas cobrando prestaciones, dado los mayores derechos a prestaciones acumulados por los indefinidos). Por último, la duración de los episodios de no empleo también se vio afectada por la crisis, aumentado desde algo menos de diez meses en 2006 a más de 12 en 2012.

\section{Tasas de salida del desempleo}

La elevada segmentación del mercado de trabajo español junto con su elevada rotación laboral (Bentolila et al., 2012) ha dado lugar a dos grupos de trabajadores. Unos tienen trayectorias laborales muy estables, donde la antigüedad observada es bastante cercana a su experiencia potencial total y por tanto, no registran interrupciones en sus carreras que puedan suponer pérdidas de capital humano. Otros, principalmente los contratados como temporales aunque a veces con algún periodo de empleo indefinido (véase Cebrian et al., 2011), presentan trayectorias inestables, su antigüedad apenas representa la mitad de su experiencia potencial y sufren recurrentes episodios de desempleo (véase Arranz y Muro, 2004).

Los Cuadros $2 \mathrm{a}$ y $2 \mathrm{~b}$ presentan las medidas de inestabilidad laboral construidas para el conjunto de trabajadores despedidos en cada uno de los cuatro años considerados. Se observa que la rotación laboral, medida por el número de contratos por año, es algo superior entre las mujeres, salvo en 2012, tiene una especial incidencia entre los jóvenes, entre los que el número medio de contratos supera ampliamente los observados entre los trabajadores de más edad, es superior entre los trabajadores de nacionalidad española y muestra una relación inversa con el nivel educativo de los trabajadores. La acusada rotación laboral del mercado de trabajo español se manifiesta también en la elevada duración de los episodios de no empleo ya que, en promedio, los trabajadores despedidos entre 2006 y 2014 solo habían pasado algo más de la mitad de su experiencia potencial total empleados. Esta medida de la intensidad de la situación de empleo es algo mayor para los hombres, crece con la edad y con el nivel educativo y nivel de cualificación de la ocupación del trabajador. Entre los trabajadores despedidos se observa además que la mayor parte de esta experiencia laboral ha tenido lugar en empleos temporales, especialmente en el caso de los jóvenes y trabajadores con menores niveles educativos.

Otra observación digna de ser destacada es que durante la crisis se redujeron las medidas de rotación laboral de los trabajadores despedidos. Esto se debe a que mientras en 2006 los trabajadores despedidos eran con mayor probabilidad aquellos más afectados por la inestabilidad laboral y que, incluso en un período de expansión económica, vivían transiciones frecuentes entre empleo y desempleo, en los años 2010 y 2012, y en menor medida también en 2014, los despidos ocurrieron de forma más generalizada entre colectivos con menor rotación laboral anterior. 


\section{CUADRO 2A}

MEDIDAS DESCRIPTIVAS DE LA INESTABILIDAD LABORAL

\begin{tabular}{|c|c|c|c|c|c|c|}
\hline & \multicolumn{3}{|c|}{2006} & \multicolumn{3}{|c|}{2014} \\
\hline & $\begin{array}{c}\text { Número } \\
\text { de empleos } \\
\text { por año }\end{array}$ & $\begin{array}{c}\text { Antigüedad } \\
\text { relativa }\end{array}$ & $\begin{array}{l}\text { Antigüedad } \\
\text { relativa } \\
\text { como } \\
\text { temporal }\end{array}$ & $\begin{array}{c}\text { Número } \\
\text { de empleos } \\
\text { por año }\end{array}$ & $\begin{array}{l}\text { Antigüiedad } \\
\text { relativa }\end{array}$ & $\begin{array}{l}\text { Antigüedad } \\
\text { relativa } \\
\text { como } \\
\text { temporal }\end{array}$ \\
\hline Hombre & 1,674 & 0,592 & 0,492 & 1,315 & 0,593 & 0,440 \\
\hline Mujer & 1,684 & 0,550 & 0,449 & 1,335 & 0,542 & 0,377 \\
\hline $16-29$ & 2,590 & 0,495 & 0,428 & 2,555 & 0,412 & 0,335 \\
\hline $30-44$ & 1,281 & 0,595 & 0,477 & 1,085 & 0,594 & 0,409 \\
\hline $45-54$ & 0,856 & 0,594 & 0,490 & 0,781 & 0,628 & 0,448 \\
\hline $55-65$ & 0,557 & 0,734 & 0,551 & 0,521 & 0,700 & 0,487 \\
\hline Nacional & 2,279 & 0,694 & 0,617 & 1,751 & 0,590 & 0,447 \\
\hline Extranjero & 1,515 & 0,540 & 0,433 & 1,194 & 0,566 & 0,405 \\
\hline No sabe leer & 2,366 & 0,539 & 0,480 & 2,310 & 0,483 & 0,403 \\
\hline Inferior al graduado & 1,823 & 0,547 & 0,476 & 1,270 & 0,564 & 0,454 \\
\hline Graduado & 1,402 & 0,569 & 0,462 & 1,215 & 0,574 & 0,417 \\
\hline Bachiller o FP & 1,384 & 0,595 & 0,449 & 1,346 & 0,579 & 0,377 \\
\hline Diplomado & 1,295 & 0,632 & 0,458 & 1,050 & 0,599 & 0,366 \\
\hline Licenciado & 1,343 & 0,649 & 0,455 & 1,145 & 0,587 & 0,355 \\
\hline Master o doctorado & 1,209 & 0,671 & 0,450 & 0,909 & 0,583 & 0,408 \\
\hline Indefinido & 0,778 & 0,724 & 0,356 & 0,747 & 0,721 & 0,303 \\
\hline Temporal & 2,112 & 0,494 & 0,453 & 1,745 & 0,480 & 0,400 \\
\hline Agricultura & 2,596 & 0,538 & 0,496 & 1,977 & 0,462 & 0,419 \\
\hline Industria & 1,293 & 0,631 & 0,456 & 0,949 & 0,650 & 0,392 \\
\hline Construcción & 1,651 & 0,585 & 0,534 & 1,004 & 0,623 & 0,496 \\
\hline Servicios de mercado & 1,776 & 0,560 & 0,443 & 1,349 & 0,573 & 0,386 \\
\hline Servicios de no mercado & 1,335 & 0,513 & 0,418 & 1,221 & 0,533 & 0,400 \\
\hline WCH & 1,057 & 0,676 & 0,422 & 0,874 & 0,701 & 0,371 \\
\hline WCL & 1,414 & 0,629 & 0,391 & 1,404 & 0,605 & 0,306 \\
\hline $\mathrm{BCH}$ & 1,780 & 0,538 & 0,382 & 1,367 & 0,576 & 0,359 \\
\hline BCL & 1,848 & 0,543 & 0,456 & 1,543 & 0,522 & 0,390 \\
\hline 11-49 empleados & 1,797 & 0,555 & 0,446 & 1,464 & 0,553 & 0,374 \\
\hline 50-499 empleados & 1,894 & 0,527 & 0,420 & 1,502 & 0,542 & 0,368 \\
\hline >500 empleados & 1,858 & 0,526 & 0,388 & 1,425 & 0,561 & 0,363 \\
\hline
\end{tabular}

FUENTE: MCVL.

NOTA: WCH son los asalariados de «cuello blanco» con mayor cualificación, WCL son los asalariados de «cuello blanco» con menor cualificación, BCH son los asalariados de «cuello azul» con mayor cualificación y los BCL son los asalariados de «cuello azul» con una menor cualificación. 
CUADRO 2B

MEDIDAS DESCRIPTIVAS DE LA INESTABILIDAD LABORAL

\begin{tabular}{|c|c|c|c|c|c|c|}
\hline & \multicolumn{3}{|c|}{2010} & \multicolumn{3}{|c|}{2012} \\
\hline & $\begin{array}{c}\text { Número } \\
\text { de empleos } \\
\text { por año }\end{array}$ & $\begin{array}{c}\text { Antigüiedad } \\
\text { relativa }\end{array}$ & $\begin{array}{l}\text { Antiguiedad } \\
\text { relativa } \\
\text { como } \\
\text { temporal }\end{array}$ & $\begin{array}{c}\text { Número } \\
\text { de empleos } \\
\text { por año }\end{array}$ & $\begin{array}{c}\text { Antigüedad } \\
\text { relativa }\end{array}$ & $\begin{array}{l}\text { Antiguiedad } \\
\text { relativa } \\
\text { como } \\
\text { temporal }\end{array}$ \\
\hline Hombre & 1,298 & 0,641 & 0,498 & 1,177 & 0,638 & 0,472 \\
\hline Mujer & 1,397 & 0,569 & 0,427 & 1,155 & 0,565 & 0,421 \\
\hline $16-29$ & 2,174 & 0,502 & 0,407 & 2,123 & 0,470 & 0,373 \\
\hline $30-44$ & 1,162 & 0,639 & 0,471 & 1,050 & 0,633 & 0,444 \\
\hline $45-54$ & 0,804 & 0,646 & 0,502 & 0,709 & 0,640 & 0,477 \\
\hline $55-65$ & 0,532 & 0,726 & 0,534 & 0,444 & 0,697 & 0,513 \\
\hline Nacional & 1,854 & 0,663 & 0,549 & 1,619 & 0,639 & 0,501 \\
\hline Extranjero & 1,167 & 0,596 & 0,444 & 1,050 & 0,600 & 0,437 \\
\hline No sabe leer & 2,335 & 0,512 & 0,436 & 2,088 & 0,510 & 0,435 \\
\hline Inferior al graduado & 1,327 & 0,596 & 0,495 & 1,140 & 0,598 & 0,490 \\
\hline Graduado & 1,174 & 0,617 & 0,469 & 1,080 & 0,611 & 0,453 \\
\hline Bachiller o FP & 1,159 & 0,626 & 0,426 & 1,142 & 0,617 & 0,410 \\
\hline Diplomado & 1,177 & 0,617 & 0,420 & 1,149 & 0,612 & 0,403 \\
\hline Licenciado & 1,145 & 0,620 & 0,397 & 1,089 & 0,623 & 0,388 \\
\hline Master o doctorado & 0,807 & 0,671 & 0,435 & 0,921 & 0,631 & 0,415 \\
\hline Indefinido & 0,733 & 0,745 & 0,336 & 0,709 & 0,747 & 0,325 \\
\hline Temporal & 1,677 & 0,531 & 0,454 & 1,624 & 0,515 & 0,430 \\
\hline Agricultura & 1,906 & 0,555 & 0,524 & 1,941 & 0,460 & 0,408 \\
\hline Industria & 0,945 & 0,690 & 0,442 & 0,897 & 0,689 & 0,416 \\
\hline Construcción & 1,089 & 0,670 & 0,562 & 0,958 & 0,680 & 0,538 \\
\hline Servicios de mercado & 1,476 & 0,590 & 0,416 & 1,305 & 0,598 & 0,410 \\
\hline Servicios de no mercado & 1,173 & 0,556 & 0,433 & 1,173 & 0,555 & 0,412 \\
\hline $\mathrm{WCH}$ & 0,930 & 0,684 & 0,401 & 0,860 & 0,705 & 0,395 \\
\hline WCL & 1,315 & 0,635 & 0,360 & 1,255 & 0,638 & 0,334 \\
\hline $\mathrm{BCH}$ & 1,443 & 0,580 & 0,358 & 1,328 & 0,589 & 0,395 \\
\hline BCL & 1,366 & 0,598 & 0,452 & 1,260 & 0,575 & 0,428 \\
\hline 11-49 empleados & 1,363 & 0,608 & 0,430 & 1,273 & 0,600 & 0,395 \\
\hline 50-499 empleados & 1,423 & 0,579 & 0,407 & 1,354 & 0,582 & 0,390 \\
\hline >500 empleados & 1,432 & 0,579 & 0,390 & 1,347 & 0,593 & 0,391 \\
\hline
\end{tabular}

FUENTE: MCVL.

NOTA: WCH son los asalariados de «cuello blanco» con mayor cualificación, WCL son los asalariados de «cuello blanco» con menor cualificación, BCH son los asalariados de «cuello azul» con mayor cualificación y los BCL son los asalariados de «cuello azul» con una menor cualificación. 
En los Gráficos 1 y 2 se presentan los resultados del análisis de la supervivencia en el desempleo a partir del estimador no paramétrico de la función de supervivencia Kaplan Meier, que tiene en cuenta la censura. En el Gráfico 1 se puede observar como la permanencia en el desempleo es mayor para los trabajadores que entraron en el desempleo en los años 2010 y 2012, como resultado de la crisis y la reducida creación de empleo en ese período. En 2014 se observa una cierta recuperación, con una menor probabilidad de supervivencia para cada duración del desempleo, aunque aún no se recuperan los valores observados en 2006. Por otra parte, se confirma, como independientemente del año de entrada, a partir de los 30 meses la probabilidad de salida del desempleo es prácticamente nula.

En el Gráfico 2 se muestran la función de supervivencia de los trabajadores en función del tipo de contrato desde el que fueron despedidos. La probabilidad de abandornar el desempleo es prácticamente igual durante los primeros ocho meses (un 5 por 100 de los desempleados salen del desempleo), independientemente del tipo de contrato. Para duraciones superiores los asalariados temporales parecen mostrar una mayor probabilidad de abandono del desempleo. No obstante, para duraciones superiores a 28 meses, la probabilidad de salida es prácticamente nula y las diferencias por tipo de contrato vuelven a ser inexistentes. Estás diferencias por tipo

\section{GRÁFICO 1 \\ FUNCIÓN DE SUPERVIVENCIA POR AÑO DE ENTRADA}

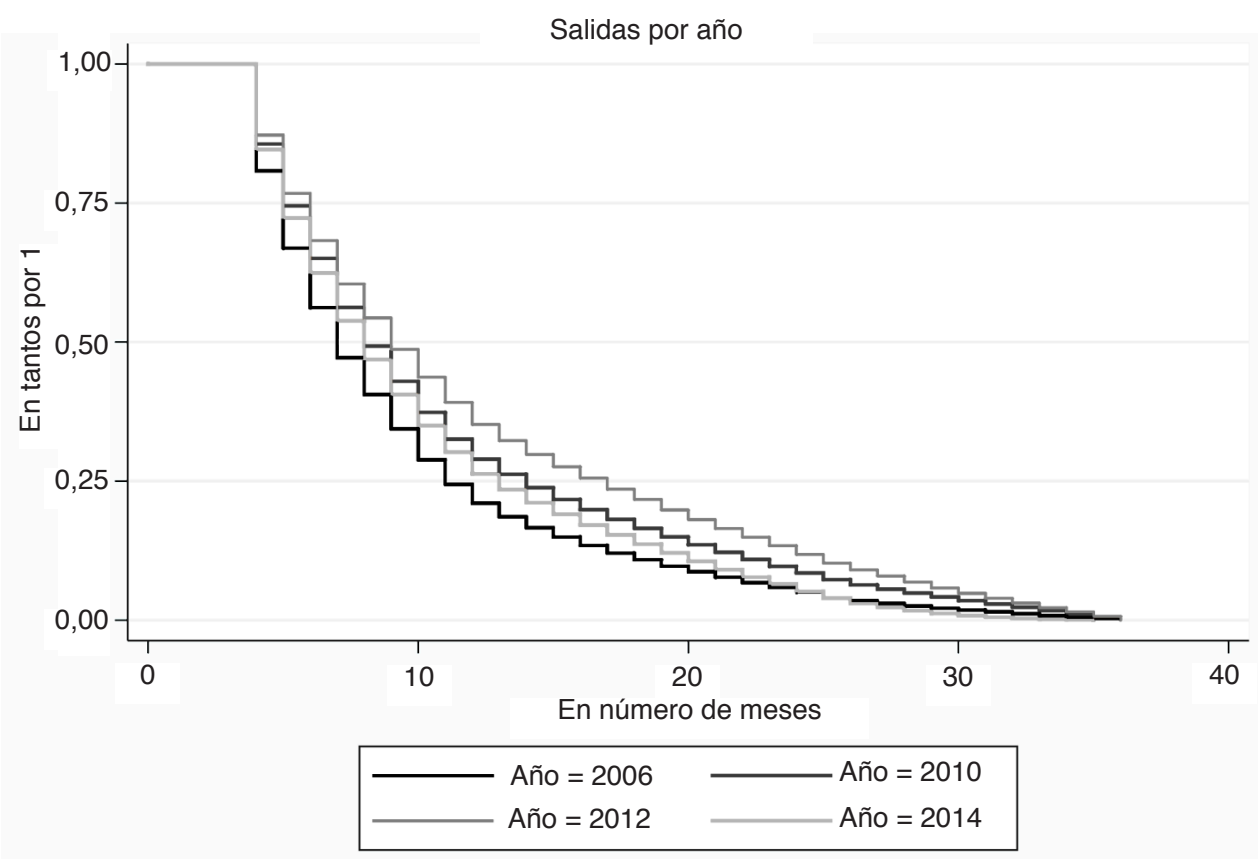

FUENTE: MCVL. 


\section{GRÁFICO 2 \\ FUNCIÓN DE SUPERVIVENCIA POR CONTRATO DE ENTRADA}

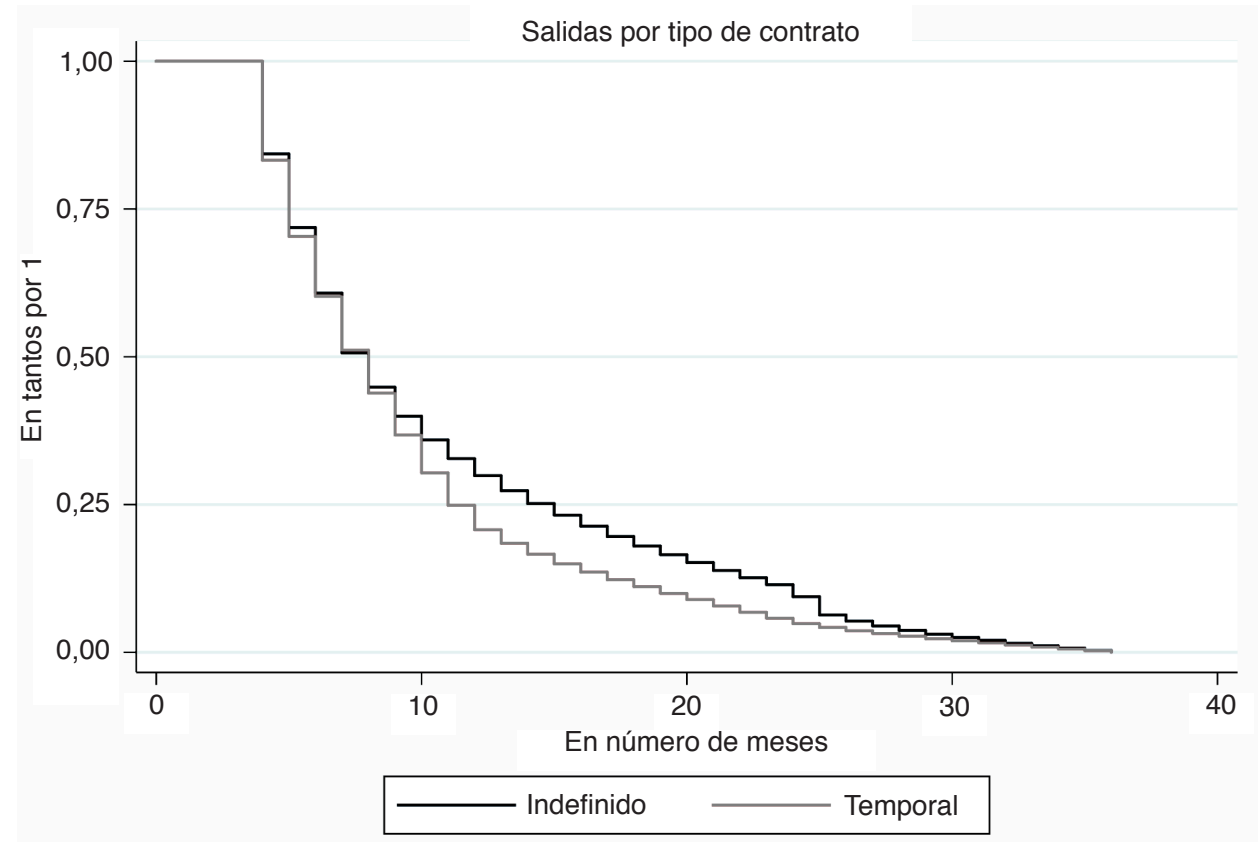

FUENTE: MCVL.

de contrato son más claras en el caso de los varones, la probabilidad de abandono del desempleo en el caso de las mujeres la superevivencia en el desempleo es mucho más similar independientemente del contrato con el que hubieran sido despedidas.

En el Cuadro 3 se presentan los resultados del modelo de Cox de riesgos proporcionales (véase Cleves et al., 2008) con especificación de la probabilidad de la permanencia en el desempleo en función de las características del trabajador (edad, sexo, nacionalidad y nivel de estudios), las medidas de su trayectoria laboral (tiempo desde que perdió el empleo, antigüedades relativas total y temporal, y número de empleos por año) y de su último empleo (ocupación, sector de actividad y tamaño de la empresa). En general, las variables explicativas son significativas y presentan el signo encontrado habitualmente en la literatura (véase, por ejemplo, García-Pérez, 1997). Así, ser mujer, tener nacionalidad extranjera, bajo nivel educativo y estar cobrando prestaciones por desempleo, se asocia a una menor probabilidad de salida del desempleo. En el caso de la variable asociada a tener un contrato temporal en el momento del despido, aunque el análisis descriptivo anterior mostraba una menor probabilidad de supervivencia para aquellos con un contrato temporal, una vez se controla por las características personales y del puesto de trabajo de origen, se observa un efecto negativo, de forma que los despedidos desde un contrato temporal permanecen durante más tiempo en el desempleo. 


\section{CUADRO 3}

MODELO DE COX. PROBABILIDAD DE PERMANENCIA EN EL DESEMPLEO (MORIR=ES SALIR) EN FUNCIÓN DE LAS CARACTERÍSTICAS DEL TRABAJADOR Y DEL ÚLTIMO EMPLEO

\begin{tabular}{|c|c|c|c|c|}
\hline & Coeficiente & T estadístico & Coeficiente & T estadístico \\
\hline Edad/100 & $-0,001$ & {$[-0,039]$} & 0,014 & {$[-0,537]$} \\
\hline Mujer & $0,026^{* * *}$ & {$[4,593]$} & $0,026 * * *$ & {$[4,564]$} \\
\hline Español & $-0,110 * * *$ & {$[-14,362]$} & $-0,112 * * *$ & {$[-14,557]$} \\
\hline $\mathrm{MES}=2$ & $-0,034 * *$ & {$[-2,401]$} & $-0,035^{* *}$ & {$[-2,433]$} \\
\hline $\mathrm{MES}=3$ & $-0,062 * * *$ & {$[-4,394]$} & $-0,062 * * *$ & {$[-4,399]$} \\
\hline $\mathrm{MES}=4$ & $-0,068 * * *$ & {$[-4,664]$} & $-0,068 * * *$ & {$[-4,663]$} \\
\hline $\mathrm{MES}=5$ & $-0,012$ & {$[-0,869]$} & $-0,012$ & {$[-0,856]$} \\
\hline $\mathrm{MES}=6$ & 0,004 & {$[0,279]$} & 0,004 & {$[0,285]$} \\
\hline $\mathrm{MES}=7$ & $-0,096 * * *$ & {$[-7,226]$} & $-0,095 * * *$ & {$[-7,179]$} \\
\hline $\mathrm{MES}=8$ & $-0,130 * * *$ & {$[-10,141]$} & $-0,129 * * *$ & {$[-10,045]$} \\
\hline $\mathrm{MES}=9$ & $-0,045 * * *$ & {$[-3,735]$} & $-0,044 * * *$ & {$[-3,673]$} \\
\hline $\mathrm{MES}=10$ & $0,168 * * *$ & {$[14,083]$} & $0,168 * * *$ & {$[14,059]$} \\
\hline MES = 11 & $0,166 * * *$ & {$[12,903]$} & $0,166 * * *$ & {$[12,897]$} \\
\hline $\mathrm{MES}=12$ & $0,079 * * *$ & {$[6,230]$} & $0,079 * * *$ & {$[6,219]$} \\
\hline Titulación inferior a graduado escolar & $0,057 * * *$ & {$[3,053]$} & $0,056 * * *$ & {$[3,002]$} \\
\hline Graduado escolar o equivalente & $0,069 * * *$ & {$[3,717]$} & $0,068 * * *$ & {$[3,620]$} \\
\hline Bachiller o formación profesional 2. ${ }^{\circ}$ grado & $0,036^{*}$ & {$[1,859]$} & $0,034 *$ & {$[1,792]$} \\
\hline Diplomado, técnico u otra titulación media & 0,038 & {$[1,635]$} & 0,036 & {$[1,555]$} \\
\hline Licenciado o graduado universitario & 0,001 & {$[0,036]$} & 0,000 & {$[-0,018]$} \\
\hline Máster, doctorado o estudios de postgrado & $-0,015$ & {$[-0,345]$} & $-0,015$ & {$[-0,358]$} \\
\hline Temporal & $0,039 * * *$ & {$[4,939]$} & $0,040 * * *$ & {$[5,082]$} \\
\hline Antigüedad relativa como indefinido & $-0,246 * * *$ & {$[-15,528]$} & $-0,258 * * *$ & {$[-15,849]$} \\
\hline Antigüedad relativa como temporal & $0,136 * * *$ & {$[11,274]$} & $0,125 * * *$ & {$[10,070]$} \\
\hline Sector: Industria & $-0,129 * * *$ & {$[-9,766]$} & $-0,134 * * *$ & {$[-10,077]$} \\
\hline Construcción & $-0,088 * * *$ & {$[-6,978]$} & $-0,093 * * *$ & {$[-7,350]$} \\
\hline Servicios de mercado & $-0,011$ & {$[-0,993]$} & $-0,016$ & {$[-1,360]$} \\
\hline Servicios no de mercado & $-0,136 * * *$ & {$[-9,758]$} & $-0,140 * * *$ & {$[-10,016]$} \\
\hline Ocupaciones: WCL & $0,037 * *$ & {$[2,126]$} & $0,037 * *$ & {$[2,119]$} \\
\hline $\mathrm{BCH}$ & $0,048 * * *$ & {$[3,400]$} & $0,048 * * *$ & {$[3,404]$} \\
\hline BCL & $0,131 * * *$ & {$[9,302]$} & $0,130 * * *$ & {$[9,292]$} \\
\hline 11 a 49 trabajadores & $0,047 * * *$ & {$[7,157]$} & $0,047 * * *$ & {$[7,153]$} \\
\hline 50 a 499 trabajadores & $0,081 * * *$ & {$[12,129]$} & $0,080 * * *$ & {$[12,076]$} \\
\hline Más de 500 trabajadores & $0,077 * * *$ & {$[8,233]$} & $0,077 * * *$ & {$[8,225]$} \\
\hline Cobrar prestaciones & & & $0,019 * * *$ & {$[3,210]$} \\
\hline Número de empleos & $-0,005^{* * *}$ & {$[-3,306]$} & $-0,004 * * *$ & {$[-2,861]$} \\
\hline Observaciones & \multicolumn{4}{|c|}{155.698} \\
\hline
\end{tabular}

FUENTE: MCVL.

NOTA: Categoría de referencia: varón, extranjero, año de despido 2006, mes de enero, no saber leer ni escribir, agricultura, trabajadores de cuellos blanco cualificados, en empresas de menos de 10 trabajadores que cobran prestaciones. WCL son los asalariados de «cuello blanco» con menor cualificación, $\mathrm{BCH}$ son los asalariados de «cuello azul» con mayor cualificación y los BCL son los asalariados de «cuello azul» con una menor cualificación. $* * * p<0,01 * * p<0,05 * p<0,1$. 
En cuanto a las variables que aproximan el impacto de la rotación laboral sobre la salida del desempleo, se observa que, una vez que se controla por un amplio conjunto de características personales y del puesto de trabajo entre las que se incluye el tipo de contrato con el que son despedidos, los trabajadores con mayor antigüedad a lo largo de toda su vida laboral como temporales presentan una menor probabilidad de salida del desempleo, lo que estaría reflejando el impacto negativo de la rotación entre contratos temporales y desempleo sobre la acumulación de capital humano de estos trabajadores. Por el contrario, los trabajadores con mayores experiencias laborales bajo un contrato indefinido presentan una mayor probabilidad de encontrar un nuevo empleo. Por ejemplo, en un caso extremo en el que un individuo tuviera toda su experiencia pasada con un contrato indefinido, su probabilidad de permanecer en el desempleo sería un 11 por 100 inferior en el promedio de los 36 meses observados tras el despido mientras que, en el caso contrario, para un individuo con toda su experiencia laboral con contratos temporales, se estima una probabilidad de permanecer en el desempleo un 18 por 100 superior.

En la segunda columna del Cuadro 3 se presentan los resultados de esta misma estimación cuando además se incluye como variable adicional el número de empleos por año del trabajador. Los resultados no cambian respecto al impacto de las variables de antigüedad como temporal o indefinido, aunque en este caso se observa un impacto negativo del número de contratos temporales sobre la probabilidad de supervivencia en el desempleo. Este resultado sugiere que el efecto de la inestabilidad laboral se produciría fundamentalmente por la menor acumulación de capital humano en el empleo y, no tanto, por depreciación de capital humano específico durante el desempleo.

\section{Pérdidas salariales por desempleo}

Los efectos negativos del desempleo relativos a la depreciación del capital humano no se reflejan exclusivamente en las tasas de salida del desempleo. Es bien conocido que el salario de reentrada en el empleo tras un periodo de desempleo es más bajo que el del empleo anterior y que dicha diferencia se amplía a medida que aumenta la duración del desempleo. Arulampalam (2001) encuentra que en el Reino Unido el salario de un individuo que reingresa en el empleo tras un periodo de desempleo de menos de un año es un 6 por 100 inferior al que recibe un trabajador similar que cambia de empleo sin experimentar ningún episodio de inactividad laboral entre ambos empleos, y que dicha diferencia puede agrandarse hasta el 14 por 100 tras cuatro años de desempleo. En el caso de Estados Unidos, Schmieder, Von Wachter y Bender (2014) estiman esta diferencia en un 0,8 por 100 por mes de desempleo. Comparando trabajadores que permanecen en el mismo empleo con los que cambian de empleo voluntaria o involuntariamente, García-Pérez y Rebollo (2005) estiman que los trabajadores que cambian involuntariamente de empleo reciben salarios en el nuevo empleo que, en comparación con los de trabajadores 
similares que permanecen en el empleo, son menores en un 8 por 100 en Portugal, 21 por 100 en Alemania, y 38 por 100 en España. Las diferencias estimadas frente a los trabajadores que cambian voluntariamente de empleo son del 14 por 100 en España y del 31 por 100 en Portugal.

La teoría del capital humano proporciona buenas razones que explican estas pérdidas salariales. La salida del empleo tiene dos consecuencias. Una es que se deja de acumular experiencia laboral y, por tanto, el capital humano general y/o específico asociado a ella. En segundo lugar, durante el desempleo el capital humano se deprecia si determinadas habilidades o aptitudes laborales empeoran por la falta de uso. Por otra parte, la búsqueda de trabajo durante los episodios de desempleo puede dar lugar a emparejamientos más o menos productivos. En la medida en que los salarios reflejan la productividad del capital humano de los trabajadores, comparaciones de salarios permiten inferir hasta qué punto estos tres factores son estadística y económicamente significativos.

A este respecto, la inestabilidad laboral en el empleo puede condicionar los efectos negativos del desempleo sobre los salarios de reentrada. Siendo cierto que hay buenas razones para esperar que los trabajadores que la sufren acumulen menos capital humano (por recibir menos formación y porque están expuestos a tareas diferentes en los diversos puestos de trabajo que ocupan), también cabe esperar que el tipo de capital humano que acumulen sea distinto al que acumulan los trabajadores que permanecen indefinidamente en un mismo puesto de trabajo. En principio, el capital humano de los primeros será más general y menos específico (a una empresa o un determinado puesto de trabajo) que los trabajadores con empleos indefinidos. Por una parte, cabe esperar que el capital humano específico se deprecia más rápidamente que el general; por otra, puede que la depreciación del capital humano se produzca más rápidamente cuando la experiencia laboral con la que se ha adquirido es más corta. Por tanto, si la inestabilidad laboral en el empleo se traduce en una mayor depreciación del capital humano y, consecuentemente, en mayores pérdidas salariales en el reempleo tras periodos de desempleo es una cuestión empírica.

En nuestra muestra obtenida a partir de datos longitudinales de la MCVL, el hecho de que los salarios de reempleo sean menores que los de salida hacia el desempleo en un periodo anterior de actividad laboral se puede comprobar fácilmente. Los Cuadros 4 y 5 , respectivamente, presentan dichos salarios para los distintos grupos de población y tipos de puestos de trabajo y el Gráfico 3 presenta las diferencias para los distintos años de la muestra. Se observa que dichas pérdidas salariales son mayores en periodos de recesión (2010 y 2012, Gráfico 3b), donde se aproximan al 15 por 100 mientras que fueron de menos del 10 por 100 en 2006 y 2014 (Gráfico 3a), y más elevadas para varones, individuos de mayor nivel educativo o con ocupaciones cualificadas, para trabajadores de la industria manufacturera y para los de las Administraciones Públicas (servicios no de mercado), para los trabajadores de mayor edad y los que tenían un contrato indefinido, y para los de empresas de 50 a 499 trabajadores. 


\section{CUADRO 4}

SALARIOS DE SALIDA DESCRIPTIVOS.

SALARIOS DEFLACTADOS PREVIOS AL DESPIDO

\begin{tabular}{|c|c|c|c|c|c|}
\hline & & 2006 & 2010 & 2012 & 2014 \\
\hline \multirow[t]{2}{*}{ Hombres } & Media & $1.403,4$ & $1.532,2$ & $1.440,9$ & $1.375,9$ \\
\hline & DT & 689,7 & 730,6 & 726,1 & 830,2 \\
\hline \multirow[t]{2}{*}{ Mujeres } & Media & $1.230,1$ & $1.436,4$ & $1.352,3$ & $1.298,3$ \\
\hline & DT & 689,5 & 723,0 & 764,0 & 757,2 \\
\hline \multirow[t]{2}{*}{ Extranjero } & Media & $1.223,6$ & $1.342,0$ & $1.215,5$ & $1.091,5$ \\
\hline & DT & 624,5 & 618,7 & 636,1 & 696,0 \\
\hline \multirow[t]{2}{*}{ Nacional } & Media & $1.337,0$ & $1.522,9$ & $1.440,2$ & $1.396,0$ \\
\hline & DT & 705,1 & 745,1 & 756,1 & 811,9 \\
\hline \multirow[t]{2}{*}{ No sabe leer } & Media & $1.186,1$ & $1.313,9$ & $1.151,3$ & $1.077,6$ \\
\hline & DT & 623,9 & 639,4 & 641,6 & 670,8 \\
\hline \multirow[t]{2}{*}{ Inferior a graduado escolar o equivalente } & Media & $1.222,0$ & $1.358,5$ & $1.238,2$ & $1.153,2$ \\
\hline & DT & 635,4 & 722,9 & 623,6 & 684,1 \\
\hline \multirow[t]{2}{*}{ Graduado escolar o equivalente } & Media & $1.291,7$ & $1.433,8$ & $1.350,8$ & $1.302,7$ \\
\hline & DT & 668,3 & 673,8 & 743,7 & 797,1 \\
\hline \multirow[t]{2}{*}{ Bachiller o FP de $2 .^{\circ}$ grado } & Media & $1.509,2$ & $1.627,5$ & $1.520,9$ & $1.473,7$ \\
\hline & DT & 758,8 & 749,4 & 737,3 & 797,4 \\
\hline \multirow[t]{2}{*}{ Diplomado o técnico } & Media & $1.626,4$ & $1.837,8$ & $1.732,4$ & $1.695,5$ \\
\hline & DT & 784,0 & 766,1 & 752,4 & 829,9 \\
\hline \multirow[t]{2}{*}{ Licenciado o graduado universitario } & Media & $1.767,8$ & $1.904,0$ & $1.818,3$ & $1.756,1$ \\
\hline & DT & 827,1 & 776,0 & 895,7 & 888,4 \\
\hline \multirow[t]{2}{*}{ Master, doctorado o estudios de postgrado } & Media & $1.961,5$ & $2.209,0$ & $1.957,2$ & $1.865,4$ \\
\hline & DT & 843,8 & 726,5 & 784,3 & 874,5 \\
\hline \multirow[t]{2}{*}{$16-29$} & Media & $1.256,0$ & $1.377,8$ & $1.251,1$ & $1.146,1$ \\
\hline & DT & 639,7 & 677,2 & 658,1 & 693,2 \\
\hline \multirow[t]{2}{*}{$30-45$} & Media & $1.323,9$ & $1.531,1$ & $1.443,1$ & $1.384,4$ \\
\hline & DT & 697,8 & 738,9 & 745,8 & 761,6 \\
\hline \multirow[t]{2}{*}{$46-54$} & Media & $1.355,5$ & $1.519,9$ & $1.464,3$ & $1.423,5$ \\
\hline & DT & 718,0 & 727,0 & 769,3 & 938,9 \\
\hline \multirow[t]{2}{*}{$55-65$} & Media & $1.510,4$ & $1.615,2$ & $1.501,1$ & $1.471,8$ \\
\hline & DT & 813,4 & 777,8 & 823,2 & 861,5 \\
\hline
\end{tabular}

FUENTE: MCVL. 
CUADRO 4 (Continuación)

SALARIOS DE SALIDA DESCRIPTIVOS. SALARIOS DEFLACTADOS PREVIOS AL DESPIDO

\begin{tabular}{|c|c|c|c|c|c|}
\hline & & 2006 & 2010 & 2012 & 2014 \\
\hline \multirow[t]{2}{*}{ Indefinido } & Media & $1.620,4$ & $1.712,0$ & $1.678,1$ & $1.734,5$ \\
\hline & DT & 774,5 & 754,1 & 734,8 & 830,3 \\
\hline \multirow[t]{2}{*}{ Temporal } & Media & $1.324,8$ & $1.472,6$ & $1.382,2$ & $1.265,8$ \\
\hline & DT & 637,5 & 656,3 & 673,0 & 726,5 \\
\hline \multirow[t]{2}{*}{ Agricultura } & Media & 733,4 & $1.168,8$ & 823,2 & 722,5 \\
\hline & DT & 532,4 & 663,6 & 498,3 & 501,8 \\
\hline \multirow[t]{2}{*}{ Industria } & Media & $1.551,3$ & $1.711,2$ & $1.679,6$ & $1.688,0$ \\
\hline & DT & 737,4 & 724,7 & 706,5 & 795,3 \\
\hline \multirow[t]{2}{*}{ Construcción } & Media & $1.350,2$ & $1.517,4$ & $1.492,1$ & $1.513,3$ \\
\hline & DT & 549,4 & 576,2 & 567,1 & 598,1 \\
\hline \multirow[t]{2}{*}{ Servicios de mercado } & Media & $1.365,4$ & $1.490,3$ & $1.461,6$ & $1.452,0$ \\
\hline & DT & 672,2 & 697,1 & 708,9 & 771,7 \\
\hline \multirow[t]{2}{*}{ Servicios de no mercado } & Media & $1.231,3$ & $1.729,8$ & $1.657,2$ & $1.561,9$ \\
\hline & DT & 733,6 & 795,1 & 760,4 & 820,6 \\
\hline \multirow[t]{2}{*}{ WCH } & Media & $2.166,8$ & $2.262,6$ & $2.122,1$ & $2.124,4$ \\
\hline & DT & 725,3 & 690,8 & 681,2 & 820,0 \\
\hline \multirow[t]{2}{*}{ WCL } & Media & $1.844,4$ & $1.934,4$ & $1.821,2$ & $1.839,8$ \\
\hline & DT & 832,5 & 785,8 & 785,8 & 888,6 \\
\hline \multirow[t]{2}{*}{$\mathrm{BCH}$} & Media & $1.375,6$ & $1.502,0$ & $1.411,7$ & $1.416,1$ \\
\hline & DT & 680,7 & 689,8 & 673,4 & 741,2 \\
\hline \multirow[t]{2}{*}{$\mathrm{BCL}$} & Media & $1.215,2$ & $1.371,9$ & $1.278,3$ & $1.204,3$ \\
\hline & DT & 631,5 & 676,1 & 706,3 & 747,2 \\
\hline \multirow[t]{2}{*}{1 a 10} & Media & $1.240,5$ & $1.387,5$ & $1.315,3$ & $1.202,1$ \\
\hline & DT & 580,2 & 592,1 & 613,4 & 659,3 \\
\hline \multirow[t]{2}{*}{11 a 49} & Media & $1.368,8$ & $1.537,9$ & $1.506,5$ & $1.414,3$ \\
\hline & DT & 646,5 & 683,6 & 705,8 & 754,5 \\
\hline \multirow[t]{2}{*}{50 a 499} & Media & $1.514,3$ & $1.714,2$ & $1.656,4$ & $1.569,7$ \\
\hline & DT & 739,2 & 889,1 & $1.014,0$ & $1.008,4$ \\
\hline \multirow[t]{2}{*}{ Más de 500} & Media & $1.270,7$ & $1.486,2$ & $1.380,6$ & $1.376,8$ \\
\hline & DT & 749,3 & 741,3 & 700,4 & 781,6 \\
\hline
\end{tabular}

FUENTE: MCVL.

NOTA: WCH son los asalariados de «cuello blanco» con mayor cualificación, WCL son los asalariados de «cuello blanco» con menor cualificación, $\mathrm{BCH}$ son los asalariados de «cuello azul» con mayor cualificación y los BCL son los asalariados de «cuello azul» con una menor cualificación. 


\section{CUADRO 5 \\ SALARIOS DEFLACTADOS DE LOS QUE SALEN DEL DESEMPLEO EN UN PERIODO POSTERIOR AL DESPIDO}

\begin{tabular}{|c|c|c|c|c|c|}
\hline & & 2006 & 2010 & 2012 & 2014 \\
\hline \multirow[t]{2}{*}{ Hombres } & Media & $1.277,2$ & $1.328,6$ & $1.212,5$ & $1.242,2$ \\
\hline & DT & 603,9 & 661,5 & 578,3 & 666,9 \\
\hline \multirow[t]{2}{*}{ Mujeres } & Media & $1.193,3$ & $1.281,6$ & $1.194,0$ & $1.210,8$ \\
\hline & DT & 627,2 & 645,9 & 560,9 & 613,4 \\
\hline \multirow[t]{2}{*}{ Extranjero } & Media & $1.141,8$ & $1.223,5$ & $1.053,0$ & $1.054,3$ \\
\hline & DT & 589,5 & 742,7 & 572,0 & 563,5 \\
\hline \multirow[t]{2}{*}{ Nacional } & Media & $1.257,9$ & $1.327,5$ & $1.237,3$ & $1.268,5$ \\
\hline & DT & 619,1 & 636,3 & 567,3 & 659,0 \\
\hline \multirow[t]{2}{*}{ No sabe leer } & Media & $1.180,1$ & $1.228,9$ & $1.052,7$ & $1.134,7$ \\
\hline & DT & 486,1 & 554,6 & 531,5 & 692,0 \\
\hline \multirow[t]{2}{*}{ Inferior a graduado escolar o equivalente } & Media & $1.188,3$ & $1.231,2$ & $1.104,2$ & $1.119,1$ \\
\hline & DT & 545,0 & 527,9 & 528,4 & 604,8 \\
\hline \multirow[t]{2}{*}{ Graduado escolar o equivalente } & Media & $1.232,3$ & $1.290,5$ & $1.200,5$ & $1.228,2$ \\
\hline & DT & 678,2 & 644,1 & 528,7 & 580,1 \\
\hline \multirow[t]{2}{*}{ Bachiller o FP de $2 .^{\circ}$ grado } & Media & $1.366,1$ & $1.397,1$ & $1.301,0$ & $1.327,6$ \\
\hline & DT & 626,0 & 710,5 & 638,4 & 693,7 \\
\hline \multirow[t]{2}{*}{ Diplomado o técnico } & Media & $1.427,9$ & $1.487,9$ & $1.410,0$ & $1.443,6$ \\
\hline & DT & 623,8 & 599,7 & 646,0 & $1.036,1$ \\
\hline \multirow[t]{2}{*}{ Licenciado o graduado universitario } & Media & $1.525,6$ & $1.580,4$ & $1.501,6$ & $1.512,1$ \\
\hline & DT & 691,8 & 708,2 & 665,0 & 680,7 \\
\hline \multirow[t]{2}{*}{ Master, doctorado o estudios de posgrado } & Media & $1.742,2$ & $1.778,0$ & $1.620,8$ & $1.657,7$ \\
\hline & DT & 706,5 & 704,4 & 674,9 & 710,8 \\
\hline \multirow[t]{2}{*}{$16-29$} & Media & $1.224,6$ & $1.282,5$ & $1.161,4$ & $1.171,6$ \\
\hline & DT & 631,0 & 572,7 & 583,9 & 628,8 \\
\hline \multirow[t]{2}{*}{$30-45$} & Media & $1.254,3$ & $1.332,5$ & $1.223,2$ & $1.252,7$ \\
\hline & DT & 585,3 & 648,3 & 572,5 & 640,2 \\
\hline \multirow[t]{2}{*}{$46-54$} & Media & $1.247,1$ & $1.309,6$ & $1.224,8$ & $1.245,9$ \\
\hline & DT & 692,1 & 896,2 & 559,4 & 664,7 \\
\hline \multirow[t]{2}{*}{$55-65$} & Media & $1.268,0$ & $1.314,9$ & $1.230,5$ & $1.269,3$ \\
\hline & DT & 523,1 & 548,2 & 527,4 & 715,4 \\
\hline
\end{tabular}

FUENTE: MCVL 


\section{CUADRO 5 (Continuación)}

\section{SALARIOS DEFLACTADOS DE LOS QUE SALEN DEL DESEMPLEO EN UN PERIODO POSTERIOR AL DESPIDO}

\begin{tabular}{|c|c|c|c|c|c|}
\hline & & 2006 & 2010 & 2012 & 2014 \\
\hline \multirow{2}{*}{ Indefinido } & Media & $1.268,7$ & $1.327,0$ & $1.263,5$ & $1.304,2$ \\
\hline & DT & 654,6 & 725,0 & 553,5 & 601,0 \\
\hline \multirow[t]{2}{*}{ Temporal } & Media & $1.236,9$ & $1.309,9$ & $1.198,9$ & $1.220,2$ \\
\hline & DT & 608,8 & 644,6 & 573,8 & 654,0 \\
\hline \multirow[t]{2}{*}{ Agricultura } & Media & 747,2 & 883,3 & 795,6 & 791,1 \\
\hline & DT & 442,4 & 493,9 & 432,1 & 451,0 \\
\hline \multirow[t]{2}{*}{ Industria } & Media & $1.255,6$ & $1.372,4$ & $1.346,2$ & $1.358,7$ \\
\hline & DT & 490,6 & 662,7 & 523,9 & 553,6 \\
\hline \multirow[t]{2}{*}{ Construcción } & Media & $1.259,9$ & $1.344,7$ & $1.319,7$ & $1.359,7$ \\
\hline & DT & 357,7 & 410,3 & 398,1 & 481,2 \\
\hline \multirow[t]{2}{*}{ Servicios de mercado } & Media & $1.235,5$ & $1.313,3$ & $1.253,4$ & $1.308,1$ \\
\hline & DT & 615,7 & 728,7 & 609,4 & 676,9 \\
\hline \multirow[t]{2}{*}{ Servicios de no mercado } & Media & $1.323,2$ & $1.312,4$ & $1.233,3$ & $1.220,1$ \\
\hline & DT & 922,9 & 588,0 & 560,6 & 731,2 \\
\hline \multirow[t]{2}{*}{ WCH } & Media & $1.848,9$ & $1.860,4$ & $1.819,1$ & $1.831,1$ \\
\hline & DT & $1.076,5$ & 675,5 & 632,1 & $1.006,8$ \\
\hline \multirow[t]{2}{*}{ WCL } & Media & $1.508,1$ & $1.527,4$ & $1.445,1$ & $1.555,0$ \\
\hline & DT & 636,8 & 611,6 & 624,6 & 674,2 \\
\hline \multirow[t]{2}{*}{$\mathrm{BCH}$} & Media & $1.226,2$ & $1.293,2$ & $1.221,8$ & $1.261,2$ \\
\hline & DT & 573,0 & 600,0 & 511,1 & 567,6 \\
\hline \multirow[t]{2}{*}{$\mathrm{BCL}$} & Media & $1.196,5$ & $1.272,1$ & $1.161,9$ & $1.181,3$ \\
\hline & DT & 569,3 & 656,0 & 560,3 & 621,3 \\
\hline \multirow[t]{2}{*}{1 a 10} & Media & $1.141,2$ & $1.216,5$ & $1.121,0$ & $1.139,9$ \\
\hline & DT & 481,3 & 520,2 & 499,5 & 546,4 \\
\hline \multirow[t]{2}{*}{11 a 49} & Media & $1.208,3$ & $1.295,0$ & $1.207,3$ & $1.238,5$ \\
\hline & DT & 531,1 & 690,9 & 516,8 & 642,8 \\
\hline \multirow[t]{2}{*}{50 a 499} & Media & $1.324,2$ & $1.405,3$ & $1.302,2$ & $1.339,9$ \\
\hline & DT & 650,3 & 669,4 & 614,8 & 686,5 \\
\hline \multirow[t]{2}{*}{ Más de 500} & Media & $1.475,2$ & $1.545,5$ & $1.451,9$ & $1.467,7$ \\
\hline & DT & 949,9 & 755,1 & 728,6 & 767,8 \\
\hline
\end{tabular}

FUENTE: MCVL.

NOTA: WCH son los asalariados de «cuello blanco» con mayor cualificación, WCL son los asalariados de «cuello blanco» con menor cualificación, $\mathrm{BCH}$ son los asalariados de «cuello azul» con mayor cualificación y los BCL son los asalariados de «cuello azul» con una menor cualificación. 


\section{GRÁFICO 3A \\ PÉRDIDAS SALARIALES TRAS DESEMPLEO (2006 Y 2014) \\ (En \%)}

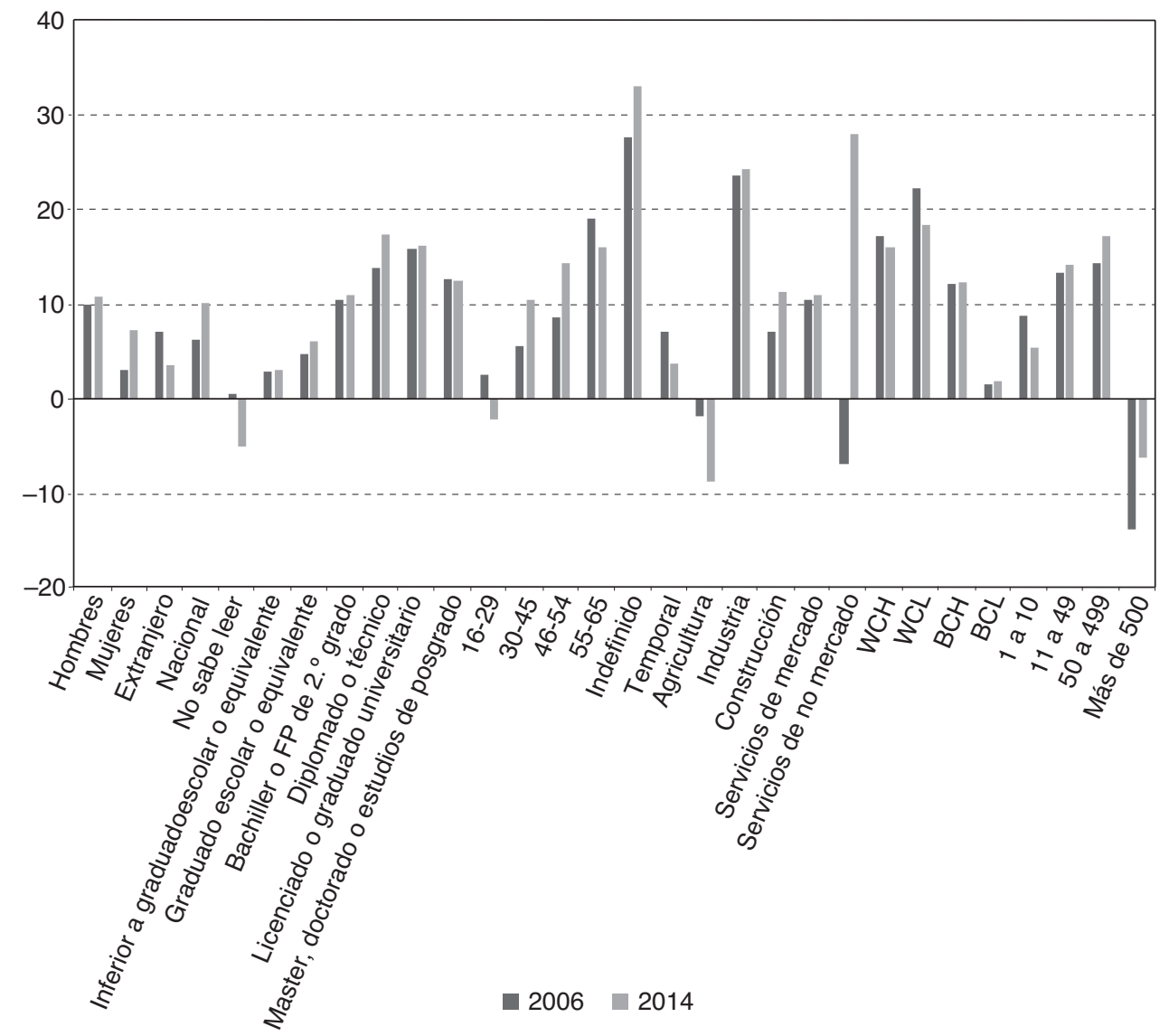

FUENTE: Elaboración propia a partir de datos de MCVL

NOTA: WCH son los asalariados de «cuello blanco» con mayor cualificación, WCL son los asalariados de «cuello blanco» con menor cualificación, BCH son los asalariados de «cuello azul» con mayor cualificación y los BCL son los asalariados de «cuello azul» con una menor cualificación.

Para documentar en qué medida la inestabilidad laboral durante periodos de empleo está asociada a la pérdida salarial que se produce por periodos posteriores de desempleo, adoptamos un enfoque standard que consiste en la estimación de ecuaciones de salarios controlando por características personales de los trabajadores y de los puestos de trabajo, añadiendo las variables que aproximan tanto la inestabilidad laboral en el periodo anterior de empleo como la duración del periodo de desempleo. Además, dado el carácter dual del mercado de trabajo español y las significativas diferencias salariales entre trabajadores indefinidos y temporales, resulta necesario 


\section{GRÁFICO 3B}

PÉRDIDAS SALARIALES TRAS DESEMPLEO (2010 Y 2012)

(En \%)

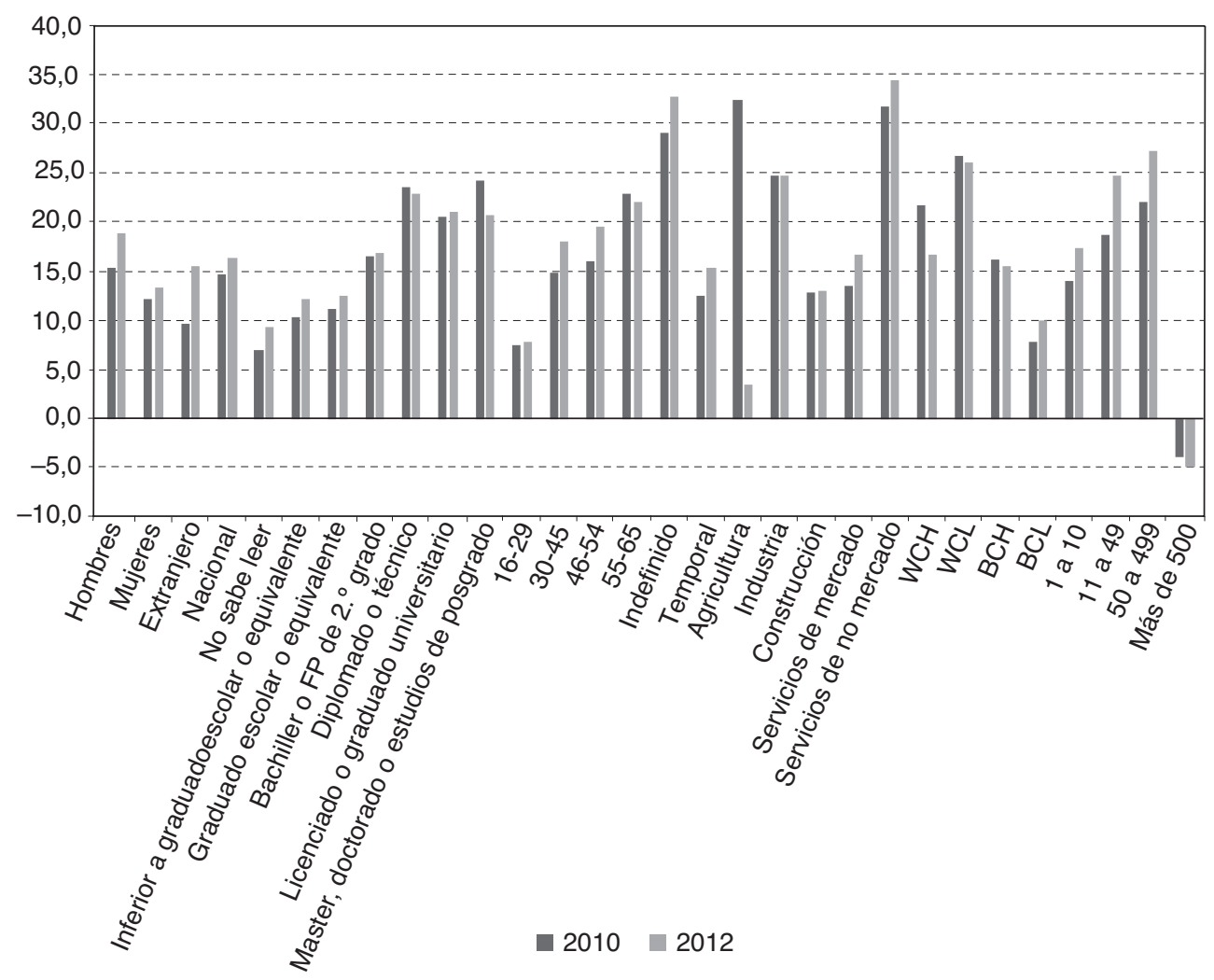

FUENTE: Elaboración propia a partir de datos de MCVL

NOTA: WCH son los asalariados de «cuello blanco» con mayor cualificación, WCL son los asalariados de «cuello blanco» con menor cualificación, $\mathrm{BCH}$ son los asalariados de «cuello azul» con mayor cualificación y los BCL son los asalariados de «cuello azul» con una menor cualificación.

controlar por la transición entre contratos de trabajo (indefinido o temporal) asociada a la pérdida de empleo y posterior reempleo.

El Cuadro 6 presenta los resultados de estas regresiones. Los resultados confirman que tanto la estabilidad como la inestabilidad laboral durante el empleo (antigüedades relativas como indefinido y como temporal), están asociadas a una mayor pérdida salarial tras un periodo de desempleo. Por ejemplo, un trabajador con un contrato indefinido que pierde su empleo y toda su trayectoria laboral anterior ha sido con contratos indefinidos sufre una pérdida salarial de entre el 20 por 100 y el 


\section{CUADRO 6}

REGRESIONES SALARIALES PARA LOS TRABAJADORES A TIEMPO COMPLETO (TANTO EN EL MOMENTO DE LA SALIDA COMO DEL NUEVO CONTRATO)

\begin{tabular}{|c|c|c|c|c|}
\hline & $\begin{array}{l}\text { Temporal- } \\
\text { temporal }\end{array}$ & $\begin{array}{l}\text { Indefinido- } \\
\text { indefinido }\end{array}$ & $\begin{array}{l}\text { Temporal- } \\
\text { indefinido }\end{array}$ & $\begin{array}{c}\text { Indefinido- } \\
\text { temporal }\end{array}$ \\
\hline \multirow[t]{2}{*}{ EDAD } & $-0,006^{* * *}$ & $-0,005^{* *}$ & $-0,006$ & $-0,024 * * *$ \\
\hline & {$[-6,305]$} & {$[-2,381]$} & {$[-1,365]$} & {$[-8,910]$} \\
\hline \multirow[t]{2}{*}{ EDAD2/100 } & $-0,007 * * *$ & $0,006 * *$ & 0,008 & $0,026 * * *$ \\
\hline & {$[5,355]$} & {$[2,423]$} & {$[1,561]$} & {$[7,597]$} \\
\hline \multirow[t]{2}{*}{ Mujer } & $0,013 * * *$ & $0,060 * * *$ & $0,034 * *$ & $0,059 * * *$ \\
\hline & {$[3,483]$} & {$[8,670]$} & {$[2,555]$} & {$[7,438]$} \\
\hline \multirow[t]{2}{*}{ Español } & $-0,053^{* * *}$ & $-0,008$ & $-0,015$ & $-0,107 * * *$ \\
\hline & {$[-10,884]$} & {$[-0,797]$} & {$[-0,857]$} & {$[-9,264]$} \\
\hline \multirow[t]{2}{*}{2010} & $-0,032 * * *$ & $-0,020 * *$ & $-0,050 * * *$ & $-0,027 * * *$ \\
\hline & {$[-7,119]$} & {$[-2,146]$} & {$[-2,854]$} & {$[-2,836]$} \\
\hline \multirow[t]{2}{*}{2012} & $-0,024 * * *$ & $-0,043 * * *$ & $-0,062 * * *$ & $-0,040 * * *$ \\
\hline & {$[-5,244]$} & {$[-4,702]$} & {$[-3,568]$} & {$[-4,060]$} \\
\hline \multirow[t]{2}{*}{2014} & $0,051 * * *$ & $-0,046 * * *$ & $-0,001$ & $-0,018$ \\
\hline & {$[11,097]$} & {$[-4,829]$} & {$[-0,046]$} & {$[-1,616]$} \\
\hline \multirow[t]{2}{*}{ Inferior a graduado escolar o equivalente } & $-0,002$ & 0,025 & $-0,004$ & $0,061 *$ \\
\hline & {$[-0,189]$} & {$[0,822]$} & {$[-0,085]$} & {$[1,831]$} \\
\hline \multirow[t]{2}{*}{ Graduado escolar o equivalente } & $-0,027 * *$ & 0,000 & $-0,021$ & 0,018 \\
\hline & {$[-2,460]$} & {$[0,006]$} & {$[-0,468]$} & {$[0,556]$} \\
\hline \multirow[t]{2}{*}{ Bachiller o FP de $2 .^{\circ}$ grado } & $-0,047 * * *$ & $-0,012$ & $-0,010$ & $-0,016$ \\
\hline & {$[-4,067]$} & {$[-0,378]$} & {$[-0,212]$} & {$[-0,483]$} \\
\hline \multirow[t]{2}{*}{ Diplomado o técnico } & $-0,074 * * *$ & $-0,020$ & $-0,089 *$ & $-0,038$ \\
\hline & {$[-5,109]$} & {$[-0,574]$} & {$[-1,688]$} & {$[-1,035]$} \\
\hline \multirow[t]{2}{*}{ Licenciado o graduado universitario } & $-0,087 * * *$ & 0,018 & $-0,065$ & $-0,031$ \\
\hline & {$[-5,924]$} & {$[0,557]$} & {$[-1,258]$} & {$[-0,837]$} \\
\hline \multirow[t]{2}{*}{ Master, doctorado o estudios de posgrado } & $-0,020$ & 0,011 & $-0,167 * *$ & $-0,184 * * *$ \\
\hline & {$[-0,647]$} & {$[0,221]$} & {$[-1,965]$} & {$[-2,716]$} \\
\hline
\end{tabular}

FUENTE: MCVL.

NOTA: $t$ estadístico entre paréntesis. $* * * p<0,01 * * p<0,05 * p<0,1$. 


\section{CUADRO 6 (Continuación)}

\section{REGRESIONES SALARIALES PARA LOS TRABAJADORES A TIEMPO COMPLETO (TANTO EN EL MOMENTO DE LA SALIDA COMO DEL NUEVO CONTRATO)}

\begin{tabular}{|c|c|c|c|c|}
\hline & $\begin{array}{c}\text { Temporal- } \\
\text { temporal }\end{array}$ & $\begin{array}{l}\text { Indefinido- } \\
\text { indefinido }\end{array}$ & $\begin{array}{l}\text { Temporal- } \\
\text { indefinido }\end{array}$ & $\begin{array}{c}\text { Indefinido- } \\
\text { temporal }\end{array}$ \\
\hline \multirow[t]{2}{*}{ Antigüedad relativa como indefinido } & $-0,128 * * *$ & $-0,130 * * *$ & $-0,039$ & $-0,239 * * *$ \\
\hline & {$[-11,192]$} & {$[-7,428]$} & {$[-1,053]$} & {$[-13,531]$} \\
\hline \multirow[t]{2}{*}{ Antigüedad relativa como temporal } & $-0,064 * * *$ & $-0,084 * * *$ & $-0,051 *$ & $-0,127 * * *$ \\
\hline & {$[-8,659]$} & {$[-3,957]$} & {$[-1,811]$} & {$[-5,585]$} \\
\hline \multirow[t]{2}{*}{ Duración del desempleo } & $0,001 * * *$ & $-0,004 * * *$ & $-0,000$ & $-0,003 * * *$ \\
\hline & {$[2,657]$} & {$[-5,821]$} & {$[-0,354]$} & {$[-5,474]$} \\
\hline \multirow[t]{2}{*}{ Industria } & $-0,009$ & $-0,126 * * *$ & 0,035 & $0,227 * * *$ \\
\hline & {$[-1,145]$} & {$[-6,881]$} & {$[0,971]$} & {$[10,022]$} \\
\hline \multirow[t]{2}{*}{ Construcción } & $0,037 * * *$ & $-0,121 * * *$ & 0,000 & $0,288 * * *$ \\
\hline & {$[5,118]$} & {$[-4,922]$} & {$[0,003]$} & {$[12,688]$} \\
\hline \multirow[t]{2}{*}{ Servicios de mercado } & $-0,009$ & $-0,163 * * *$ & $-0,054^{*}$ & $0,202 * * *$ \\
\hline & {$[-1,363]$} & {$[-9,944]$} & {$[-1,722]$} & {$[9,473]$} \\
\hline \multirow[t]{2}{*}{ Servicios de no mercado } & $-0,006$ & $-0,116^{* * *}$ & $-0,065$ & $0,223 * * *$ \\
\hline & {$[-0,742]$} & {$[-5,372]$} & {$[-1,592]$} & {$[9,033]$} \\
\hline \multirow[t]{2}{*}{ WCL } & $-0,053 * * *$ & $-0,108 * * *$ & $-0,119$ *** & $-0,071 * * *$ \\
\hline & {$[-3,798]$} & {$[-6,534]$} & {$[-3,489]$} & {$[-3,014]$} \\
\hline \multirow[t]{2}{*}{$\mathrm{BCH}$} & $-0,116^{* * *}$ & $-0,152 * * *$ & $-0,227 * * *$ & $-0,154 * * *$ \\
\hline & {$[-11,822]$} & {$[-10,444]$} & {$[-8,495]$} & {$[-8,743]$} \\
\hline \multirow[t]{2}{*}{$\mathrm{BCL}$} & $-0,059$ *** & $-0,080 * * *$ & $-0,203^{* * *}$ & $-0,059 * * *$ \\
\hline & {$[-6,090]$} & {$[-5,391]$} & {$[-7,484]$} & {$[-3,297]$} \\
\hline \multirow[t]{2}{*}{11 a 49} & 0,003 & 0,007 & $-0,011$ & $-0,002$ \\
\hline & {$[0,674]$} & {$[0,775]$} & {$[-0,705]$} & {$[-0,231]$} \\
\hline \multirow[t]{2}{*}{50 a 499} & $0,031 * * *$ & 0,008 & 0,020 & $0,049 * * *$ \\
\hline & {$[7,451]$} & {$[0,932]$} & {$[1,231]$} & {$[5,465]$} \\
\hline \multirow[t]{2}{*}{ Más de 500} & $0,060 * * *$ & 0,011 & $0,046^{*}$ & $0,113 * * *$ \\
\hline & {$[10,641]$} & {$[0,907]$} & {$[1,935]$} & {$[8,873]$} \\
\hline \multirow[t]{2}{*}{ Constante } & $0,210 * * *$ & $0,320 * * *$ & $0,275 * * *$ & $0,417 * * *$ \\
\hline & {$[9,060]$} & {$[5,567]$} & {$[2,960]$} & {$[6,485]$} \\
\hline Número de observaciones & 103.109 & 16.917 & 6.491 & 17.305 \\
\hline $\mathrm{R}$ cuadrado & 0,015 & 0,035 & 0,023 & 0,069 \\
\hline
\end{tabular}

FUENTE: MCVL.

NOTA: WCH son los asalariados de «cuello blanco» con mayor cualificación, WCL son los asalariados de «cuello blanco» con menor cualificación, BCH son los asalariados de «cuello azul» con mayor cualificación y los BCL los asalariados de «cuello azul» con una menor cualificación.

$t$ estadístico entre paréntesis. $* * * p<0,01 * * p<0,05 * p<0,1$. 
33 por 100 en el empleo posterior dependiendo de si este es con un contrato temporal o indefinido, respectivamente. En el caso de un trabajador con un contrato temporal, con experiencia laboral únicamente como temporal estas mismas cifras son del 9,8 por 100 y 13,2 por 100, respectivamente. En el primer caso, la justificación es la mayor pérdida de capital humano específico que se acumula en puestos de trabajo indefinidos dentro de la misma empresa. En el segundo caso, las pérdidas salariales son cuantitativamente menores, pero no por ello irrelevantes. Dado que pueden achacarse en menor medida a la pérdida de capital humano específico cabe pensar que se originan por una mayor tasa de depreciación del capital humano general adquirido en puestos de trabajo diversos. No obstante, esta es una conjetura que debería ser confirmada en futuros trabajos de investigación.

\section{Comentarios finales}

La dualidad contractual en el mercado de trabajo español tiene consecuencias en relación con transiciones laborales y diferencias salariales que han sido ampliamente estudiadas y diagnosticadas. Entre ellas la relación entre transiciones laborales y capital humano acumulado por los trabajadores es de especial importancia en la medida en que determina sus ganancias de productividad a través de la experiencia laboral. Documentar la magnitud de este efecto es una tarea complicada por las dificultades de medición de la productividad y de identificación de los efectos causales del tipo de contratación laboral sobre oportunidades futuras de empleo y salarios.

En este trabajo hemos realizado una primera aproximación a esta cuestión estimando la asociación entre inestabilidad laboral, por una parte, y tasas de salida hacia el empleo tras un periodo posterior de desempleo y salarios en el nuevo empleo, por otra. Los resultados muestran que las tasas de salida del desempleo son tanto menores cuanto más inestable sea el periodo previo de empleo. También encontramos que, si bien las pérdidas salariales desde trayectorias estables de empleo son mayores, la inestabilidad laboral también está asociada a un menor salario en el reempleo, incluso a pesar de que la depreciación de capital humano específico en estas trayectorias sea menos acusada.

Estos resultados tienen implicaciones directas sobre el debate acerca de la conveniencia de eliminar la dualidad contractual en el mercado de trabajo español con el objetivo de reducir la inestabilidad laboral en el empleo. A efectos del diseño y la implementación de políticas de empleo, sugieren, por ejemplo, que las trayectorias laborales previas deberían ser tenidas en cuenta a la hora de decidir qué tipo de formación en el empleo y en el desempleo se ofrece y el perfilado de los trabajadores desempleados en la búsqueda de un nuevo empleo. 


\section{Referencias bibliográficas}

[1] ALBERT, C.; GARCÍA-SERRANO, C. y HERNANZ, V. (2005). «Firm-provided training and temporary contracts». Spanish Economic Review, 7 (1), 67-88.

[2] ARRANZ, J. M.; GARCÍA-SERRANO, C. y HERNANZ, V. (2013). «How do we pursue "labormetrics"? An application using the MCVL». Estadística Española, 55 (181), 231-254.

[3] ARRANZ, J. M. y MURO, J. (2004). «Recurrent unemployment, welfare benefits and heterogeneity». International Review of Applied Economics, 18 (4), 423-441.

[4] ARRAZOLA, M.; DE HEVIA, J.; RISUEÑO, M. y SANZ, J. F. (2005). «A proposal to estimate human capital depreciation: Some evidence for Spain». Hacienda Publica Española-Revista de Economia Publica, 172 (1), 9-22.

[5] ARULAMPALAM, W. (2001). «Is unemployment really scarring? Effects of unemployment experiences on wages». The Economic Journal, 111 (475), 585-606.

[6] BENTOLILA, S.; CAHUC, P.; DOLADO, J. J. y LE BARBANCHON, T. (2012). «Two-tier labour markets in the Great Recession: France versus Spain». The Economic Journal, 122 (562), F155-F187.

[7] CABRALES, A.; DOLADO, J. y MORA, R. (2014). «Dual labour markets and (lack of) on-the-job training: PIAAC evidence from Spain and other EU countries». SERIEs: Journal of the Spanish Economic Association, 8 (4), 345-371, november.

[8] CEBRIÁN, I.; MORENO, G. y TOHARIA, L. (2011). «La estabilidad laboral y los programas de fomento de la contratación indefinida». Hacienda Pública Española, 3 (198), 103-127.

[9] CLEVES, M.; GOULD, W.; GOULD, W. W.; GUTIERREZ, R. y MARCHENKO, Y. (2008). An introduction to survival analysis using Stata. College Station, Texas. Stata Press Publication StataCorp LP.

[10] EDIN, P. A. y GUSTAVSSON, M. (2008). «Time out of work and skill depreciation». ILR Review, 61 (2), 163-180.

[11] DE LA FUENTE, A. y JIMENO, J. F. (2009). «The Private and Fiscal Returns to Schooling in the European Union». The Journal of the European Economic Association, 7, 6, 1319-1360.

[12] FELGUEROSO, F. (2015). «Claves para mejorar la educación y formación de adultos en España en la poscrisis». Fundación de Estudios de Economía Aplicada (FEDEA). Estudios sobre la Economía Española, 2015/12.

[13] GARCÍA-PÉREZ, J. I. (1996). «Las tasas de salida del empleo y el desempleo en España (1978-1993)». Investigaciones Económicas, 21 (1), 29-53, enero 1997.

[14] GARCÍA-PÉREZ, J. I.; MARINESCU, I. y VALL-CASTELLO, J. (2018): «Can FixedTerm Contracts Put Low Skilled Youth on a Better Career Path? Evidence from Spain». Forthcoming in The Economic Journal.

[15] GARCÍA-PÉREZ, J. I. y REBOLLO-SANZ, Y. F. (2005). «Wage changes through job mobility in Europe: A multinomial endogenous switching approach». Labour Economics, 12 (4), 531-555.

[16] GREGORY, M. y JUKES, R. (2001). «Unemployment and subsequent earnings: Estimating scarring among British men 1984-94». The Economic Journal, 111 (475), 607-625. 
[17] JENKINS, S. P. y GARCÍA-SERRANO, C. (2004). «The relationship between unemployment benefits and re-employment probabilities: evidence from Spain». Oxford Bulletin of Economics and Statistics, 66 (2), 239-260.

[18] MINCER, J. (1974). Schooling, Experience and Earnings. Nueva York: National Bureau of Economic Research.

[19] MINCER, J. y OFEK, H. (1982). «Interrupted work careers: Depreciation and restoration of human capital». Journal of Human Resources, 3-24.

[20] PERAITA, C. (2005). «Firm-sponsored training in regulated labour markets: evidence from Spain». Applied Economics, 37 (16), 1885-1898.

[21] REBOLLO-SANZ, Y. (2012). «Unemployment insurance and job turnover in Spain». Labour Economics, 19 (3), 403-426.

[22] REBOLLO-SANZ, Y. F. y GARCÍA-PÉREZ, J. I. (2015). «Are unemployment benefits harmful to the stability of working careers? The case of Spain». SERIEs, 6 (1), 1-41.

[23] SCHMIEDER, J. F.; VON WACHTER, T. y BENDER, S. (2016). «The effect of unemployment benefits and nonemployment durations on wages». American Economic Review, 106 (3), 739-77. 